\title{
Environmental and economic comparatives assessments of wine making processes: Application to the enrichment of wines
}

\author{
Sophie Penavayre ${ }^{1}$, Valérie Lempereur ${ }^{1}$, Peio Elichiry-Ortiz ${ }^{1}$, Philippe Cottereau ${ }^{2}$, et Denis Caboulet ${ }^{3}$ \\ ${ }^{1}$ Institut Français de la Vigne et du Vin (IFV), Pôle Bourgogne-Beaujolais-Savoie-Jura, 69661 Villefranche-sur-Saône, France \\ ${ }^{2}$ Institut Français de la Vigne et du Vin (IFV), Pôle Rhône-Méditerranée, 7 avenue Yves Cazeaux, 30230 Rodilhan, France \\ ${ }^{3}$ Institut Français de la Vigne et du Vin (IFV), Pôle Rhône-Méditerranée, Domaine de Pech Rouge, 11430 Gruissan, France
}

\begin{abstract}
The project Enrichment, managed by the French Institute for Vine and Wine (IFV) from 2013 to 2015, aimed to joint assessments of the potential environmental impacts and the costs for the cellars of two new enrichment methods, compared to methods commonly used. A "life cycle" approach has been chosen. Actually, the potential environmental impacts has been assessed following the recommendations from the normalized method of the Life Cycle Assessment (LCA). The method of the Life Cycle Cost Assessment (LCCA) has been linked to the LCA in order to assess the costs of the different techniques. The method and the developed tool seem adapted to achieve to goals of the project: the comparison of environmental and economic impacts of the enrichment of $1 \mathrm{hl}$ of must by $1 \%$ vol. using different techniques, whether additive or subtractive. Beyond, the project gives a framework for the environmental and economic comparison of different techniques for any other industrial process.
\end{abstract}

\section{Introduction}

La conception d'outils, de méthodes, ou encore de procédures, nécessite d'évaluer la faisabilité technique et organisationnelle de l'objet conçu. Le coût de la conception et de l'utilisation de l'objet est également estimé. La généralisation de l'éco-conditionnalité (i.e. subordonner ses achats au respect de principes et critères environnementaux) dans le domaine des achats mais également dans celui des aides publiques, amène le besoin d'une évaluation des impacts de l'objet étudié sur les ressources naturelles et plus généralement sur l'environnement. Cette évaluation des impacts environnementaux doit alors pouvoir être réalisée a priori i.e. au moment de la conception de l'objet.

La méthode de l'Analyse de Cycle de Vie (ACV) répond à ce besoin. Il s'agit d'une méthode d'évaluation des impacts environnementaux potentiels d'un bien ou d'un service. L'ACV est multi-étapes (étudie les impacts de la fabrication d'un produit tout au long de son cycle de vie, de la conception à la gestion des déchets, en passant par la fabrication et la phase d'utilisation) et multi-critères (exprime les dommages potentiels sur la santé humaine, les écosystèmes ou encore les ressources naturelles). Elle peut être utilisée pour évaluer un objet au moment de sa conception, avant même sa mise en œuvre. Utilisée pour des évaluations comparatives, elle permet d'identifier les points critiques et d'évaluer les impacts d'une modification de l'objet, tout en veillant à éviter les transferts de pollutions (méthode multi-critères). Développée au début des années 1990 pour les besoins de l'industrie de la chimie, l'ACV a depuis été appliquée à d'autres secteurs, notamment agroalimentaires, dont le secteur vinicole. L'ACV dispose d'une série de normes définissant ses principes et les conditions de son utilisation (ISO 14040 à 14044 [1]).

Développée en suivant les principes de l'ACV, la méthode plus récente de l'Analyse des Coûts du Cycle de Vie (ACCV) entend évaluer les coûts de l'objet étudié [2]. Les données nécessaires à la réalisation d'une ACV et d'une ACCV sont proches : alors que la première nécessite d'identifier les flux physiques entrants et sortants à chaque étape du cycle de vie de l'objet (ex : quantité d'eau consommée pour la fabrication, quantité de déchets produits lors de la phase d'utilisation...), la seconde se base sur les flux financiers intervenants sur l'ensemble du cycle de vie (ex : remboursement d'un prêt bancaire lié à un investissement pour la fabrication, facture d'électricité consommée lors de la phase d'utilisation...).

Le couplage des méthodes ACV et ACCV pourrait alors permettre l'évaluation environnementale et économique d'un objet au moment de sa conception. ACV et ACCV couplées semblent ainsi répondre en partie aux objectifs du projet Enrichissement.

L'enrichissement consiste à augmenter la teneur en sucres du moût, par ajout de sucres fermentescibles ou par retrait de l'eau des grappes ou du moût. Ces techniques entraînent une hausse du titre alcoométrique volumique probable, TAVP, et ainsi du degré final des vins (titre alcoométrique volumique, TAV). La nécessité d'enrichir dépend de la maturité des raisins et est étroitement liée aux conditions météorologiques du millésime. La pratique de l'enrichissement est très encadrée sur le plan réglementaire tant communautaire que Français [3]. 
Celui-ci s'inscrit dans le contexte de la réforme de l'OCM vitivinicole de 2008 qui a mis fin au dispositif d'aides à l'utilisation de moût concentré et de moût concentré rectifié à partir de la campagne 2012 [4]. A la demande des professionnels concernés par la fin de la mesure, une réflexion a été engagée par les pouvoirs publics français. Une expertise des différentes voies alternatives a souligné la nécessité d'encourager le développement de deux nouvelles méthodes d'enrichissement des vins : l'utilisation du sous-produit issu du traitement œnologique consistant en la réduction de la teneur en sucre des moûts (rétentat de RTS), et l'utilisation d'un nouveau produit qualifié de « moût concentré de qualité » obtenu par électrodialyse (ajout de MCQ).

Le projet Enrichissement, coordonné par l'Institut Français de la Vigne et du Vin (IFV) de 2013 à 2015, a pour objectif l'évaluation des deux nouvelles méthodes d'enrichissement sur les critères de la faisabilité technique, du coût pour l'opérateur et de l'impact environnemental. Les volets économique et environnemental sont traités conjointement, via une ACV et une ACCV comparatives couplées. En effet, les impacts environnementaux potentiels et les coûts pour la cave des deux nouvelles méthodes d'enrichissement sont évalués en comparaison aux méthodes habituellement utilisées (ajout de saccharose, ajout de Moût Concentré Rectifié-MCR et auto-enrichissement par osmose inverse-OI).

Au-delà de l'évaluation des impacts environnementaux et des coûts des différentes techniques d'enrichissement, l'objectif du projet est de proposer une méthode et un outil permettant d'évaluer des points de vue économiques et environnementaux des itinéraires de vinification, voire tout itinéraire de transformation agroalimentaire.

\section{Matériels et méthode}

L'évaluation environnementale et économique a été réalisée en suivant les principes de l'ACV et de l'ACCV. L'étude s'est déroulée en quatre étapes : la définition des objectifs et des frontières du système étudié, la constitution de l'Inventaire de Cycle de Vie (ICV), l'analyse environnementale et économique, l'interprétation des résultats.

\subsection{Techniques d'enrichissement}

Parmi les cinq techniques d'enrichissement étudiées, trois sont autorisées : l'ajout de saccharose, l'ajout de moût concentré rectifié (MCR) et l'Osmose Inverse (OI). Les conditions d'ajout de saccharose dépendent de la région viticole [3]. La méthode d'élaboration du MCR et sa composition sont encadrées [5]. L'OI fait partie des techniques soustractives d'enrichissement utilisables sur moût [6].

Pour les deux nouvelles méthodes à l'étude, le moût concentré de qualité, MCQ, et le retentât obtenu après réduction de la teneur en sucre, nommé retentât de RTS, les hypothèses d'élaboration de ces produits ont été définies sur la base des expérimentations réalisées dans le cadre du volet faisabilité technique et organisationnelle du projet Enrichissement. Le MCQ est élaboré à partir de raisins blancs ou de raisins rouges, dont le moût est filtré, stabilisé par électrodialyse puis transporté jusqu'à une distillerie

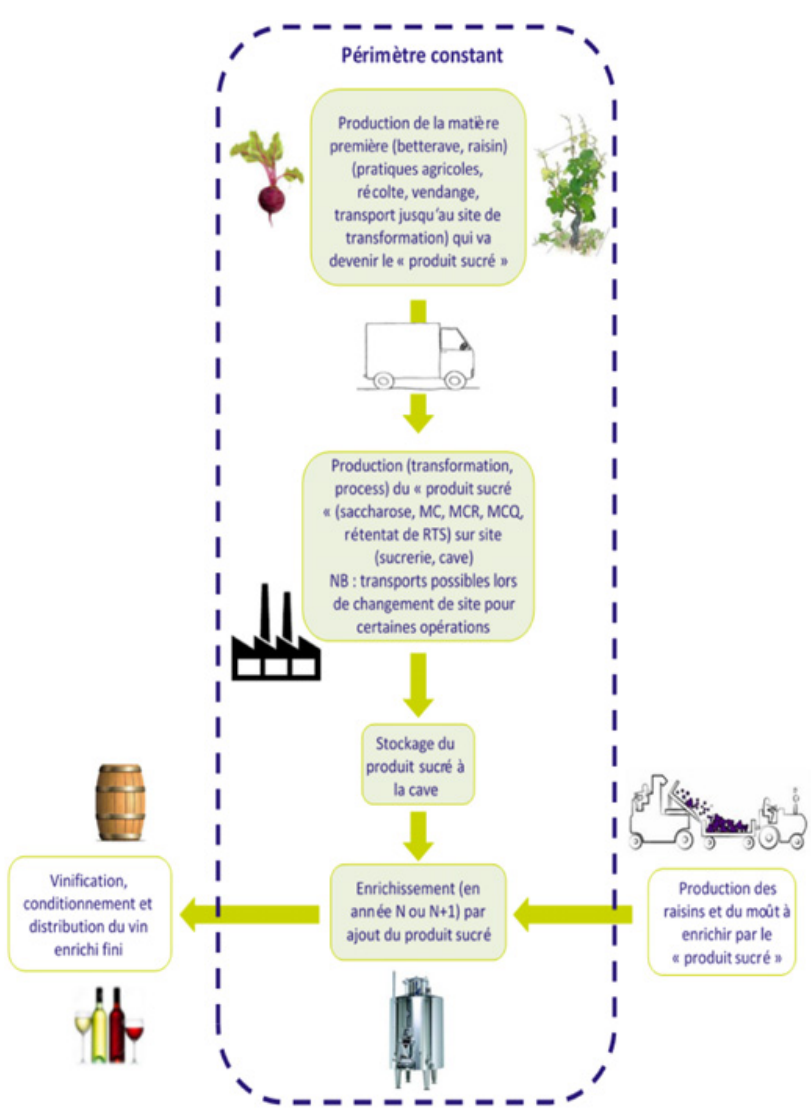

Figure 1. Périmètre défini pour l'évaluation environnementale et économique comparée des différentes techniques d'enrichissement.

afin d'y être concentré par évaporation sous vide. Il est produit l'année $\mathrm{N}$ puis stocké en vue de son utilisation en année $\mathrm{N}+1$. Pour l'élaboration de MCQ rouge, les raisins rouges subissent une étape de thermovinification suivie d'un pressurage puis d'un débourbage. La teneur en sucre du MCQ s'élève à environ $800 \mathrm{~g} / 1$.

Le retentât de RTS est le retentât obtenu après la technique de desucrage des moûts par technique membranaire. Le rétentat de RTS est quasi incolore, sa teneur en sucre s'élève à environ $400 \mathrm{~g} / \mathrm{l}$.

\subsection{Périmètre de l'étude}

L'étude ayant un but comparatif, les frontières du système étudié (i.e. le périmètre) doivent être constantes entre les différentes techniques étudiées. Le périmètre retenu est : de la fabrication du produit sucré (saccharose, MCR, $\mathrm{MCQ}$, rétentat de RTS) à son utilisation pour enrichir. La production du moût (viticulture, récolte et pressurage) ainsi que les étapes suivant l'enrichissement (préparation au conditionnement, conditionnement, distribution, consommation...) sont exclues du périmètre d'étude (cf. Fig. 1).

\subsection{Unité fonctionnelle}

L'ACV évalue les impacts environnementaux potentiels de la fonction d'un produit ou d'un service. En effet, un produit pouvant avoir plusieurs fonctions, il est important 
Tableau 1. Caves types étudiées.

\begin{tabular}{|l|c|c|c|c|}
\hline Critères & $\begin{array}{l}\text { Sud > } \\
\mathbf{2 0 ~ 0 0 0 ~ h l}\end{array}$ & $\begin{array}{l}\text { Sud }< \\
\mathbf{2 0} 000 \text { hl }\end{array}$ & $\begin{array}{l}\text { Nord }> \\
\mathbf{2 0 0 0 0} \text { hl }\end{array}$ & $\begin{array}{l}\text { Nord }< \\
\mathbf{2 0 0 0 0 ~ h l}\end{array}$ \\
\hline $\begin{array}{l}\text { Nombre de salariés } \\
\text { pendant les } \\
\text { vinifications }\end{array}$ & 24 & 3 & 16 & 2 \\
\hline $\begin{array}{l}\text { Temps horaire } \\
\text { journalier pendant } \\
\text { les vinifications (h) }\end{array}$ & 8 & 8 & 8 & 8 \\
\hline $\begin{array}{l}\text { Surface en } \\
\text { production (ha) }\end{array}$ & 1050 & 30 & 520 & 9.6 \\
\hline $\begin{array}{l}\text { Volume total } \\
\text { produit (hl) }\end{array}$ & 80000 & 18000 & 25900 & 620 \\
\hline $\begin{array}{l}\text { Distance entre la } \\
\text { cave et la distillerie } \\
\text { (km) }\end{array}$ & 10 & - & 400 & - \\
\hline $\begin{array}{l}\text { Distance moyenne } \\
\text { entre la cave et les } \\
\text { prestataires (km) }\end{array}$ & 50 & 50 & 50 & 50 \\
\hline
\end{tabular}

de définir celle qui est à l'étude. Une Unité Fonctionnelle (UF) doit donc être définie. L'UF choisie pour le projet Enrichissement rend compte du service rendu par chaque méthode d'enrichissement : enrichir 1 hl de moût de $1 \%$ vol.

\subsection{Caves types}

Les flux physiques, les coûts, les techniques d'enrichissement autorisées ... varient en fonction de la configuration de la cave (localisation, taille, types de produits ...). Le caractère comparatif de l'étude nécessite d'étudier chaque technique au sein d'une même cave. Afin de prendre en compte à la fois les réalités de caves produisant des volumes plus ou moins importants, et les contraintes liées à la règlementation (les techniques autorisées pour l'enrichissement des vins varient en fonction des zones viticoles), 4 caves types ont été définies. Deux caves se situent dans un bassin de production viticole situé au nord de la France, bassin « nord », où l'ajout de saccharose est autorisé ; les deux autres caves se situent dans un bassin de production viticole situé au sud de la France, bassin 《sud », où la technique habituellement employée pour l'enrichissement des vins est l'ajout de MCR. Dans chaque région, une cave produit moins de $20000 \mathrm{hl}$ et l'autre produit plus de $20000 \mathrm{hl}$ de vin. Les critères définis pour chaque cave-type sont (pour une année) : le nombre de salariés pendant les vinifications et la durée de travail quotidienne, la surface en production, le volume total de vin produit et le pourcentage de vin rouge et de vin blanc ou rosé, la distance entre la cave et les prestataires intervenant dans le process (cf. Tableau 1).

\subsection{Hypothèses}

Les processus mis en œuvre pour chacune des techniques étudiées ont été dessinés précisément. Des hypothèses générales sont définies. Elles concernent le TAVP des moûts à enrichir (11\% vol.) et le TAVP des vins enrichis (12\% vol.) ; les concentrations finales en sucre du MCR, du MCQ (800 g/l) et du rétentat de RTS (400 g/l) ; le niveau d'équipement des caves (seul l'achat des équipements nécessaires à l'enrichissement par les deux nouvelles méthodes est pris en compte) ; ou encore le volume de

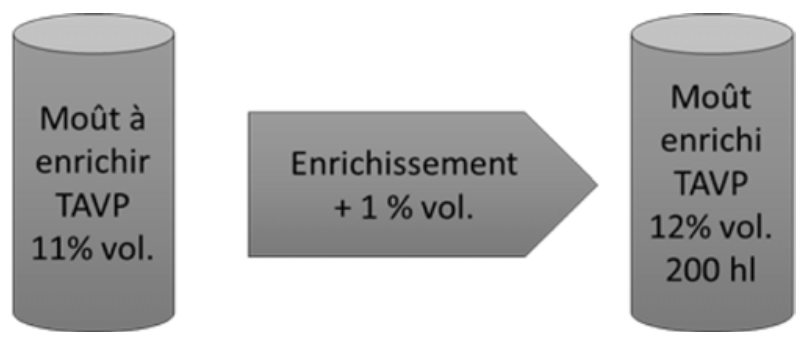

Figure 2. Hypothèse préalable à l'étude.

moût enrichi (quelles que soit la cave type ou la méthode d'enrichissement, on considère un volume de $200 \mathrm{hl}$ de moût enrichi) (cf. Fig. 2). Afin de comparer les charges environnementales et économiques supportées par la cave pour les différentes techniques, l'hypothèse que chaque cave élabore son propre MCQ et rétentat de RTS, à partir de sa vendange, a été formulée.

Deux modèles économiques différents ont été définis afin de prendre en compte les différences induites par le choix d'internaliser ou d'externaliser certaines opérations, notamment liées à la fabrication du produit sucré.

Des scenarii sont définis en regroupant une méthode d'enrichissement (ex : ajout de MCQ), une cave type (ex : cave produisant plus de $20000 \mathrm{hl}$, bassin «sud ») et un modèle économique (ex : prestation). 36 scenarii ont ainsi été étudiés.

\subsection{Construction des Inventaires de Cycle de Vie}

Un ICV environnemental et un ICV économique sont établis pour chaque scenario, en suivant le périmètre prédéfini : de la fabrication du produit sucré à son utilisation pour enrichir.

Pour la phase viticole (production des raisins qui seront transformés en MCQ, en rétentat de RTS ou en moût enrichi par Osmose Inverse), l'ICV environnemental a été constitué à l'aide des ICV présents dans la base de données AGRIBALYSE ${ }^{\circledR}$ [7]. Cette base de données d'ICV contient en effet deux ICV répondants aux besoins de l'étude : un ICV de production de raisins de cuve selon un itinéraire AOP, dans la région du Beaujolais, et un ICV de production de raisins de cuve selon un itinéraire IGP, dans la région Languedoc-Roussillon. L'ICV économique a été établi à partir de données de coûts de production définis par FranceAgriMer dans le cadre de l'Observatoire viticole 2009-2010 [8] : $3995 €$ /ha pour le bassin «sud» (avec un rendement de $60 \mathrm{hl} / \mathrm{ha}$ ) et $6775 €$ /ha pour le bassin « nord 》 (avec un rendement de $64 \mathrm{hl} / \mathrm{ha}$ ).

Pour les phases de transformation du raisin en produit sucré et d'enrichissement, les ICV ont été construits à partir de données collectées auprès d'experts. Les quantités de flux entrants et sortants pour chaque étape constituent l'ICV environnemental : consommations d'énergie et d'eau, intrants œnologiques et de nettoyage, déchets... L'ICV économique a été construit conjointement : identification des coûts liés aux charges fixes (acquisition de matériels et maintenance) et aux charges variables (consommations d'énergie et d'eau, achats d'intrants ....).

Les ICV de l'enrichissement par ajout de saccharose et de MCR ont été construits différemment. L'ICV environnemental de l'enrichissement par ajout de saccharose 
Tableau 2. Indicateurs d'impacts retenus.

\begin{tabular}{|l|l|l|}
\hline Indicateur & $\begin{array}{l}\text { Méthode de } \\
\text { caractérisation }\end{array}$ & Description \\
\hline $\begin{array}{l}\text { Changement } \\
\text { climatique }\end{array}$ & Impact 2002+ & $\begin{array}{l}\text { Potentiel d'effet de serre additionnel } \\
\text { engendré par le cycle de vie }\end{array}$ \\
\hline Ressources & Impact 2002+ & $\begin{array}{l}\text { Quantité totale des énergies fossiles et } \\
\text { minérales consommées au cours du cycle } \\
\text { de vie }\end{array}$ \\
\hline $\begin{array}{l}\text { Eutrophisation } \\
\text { des eaux douces }\end{array}$ & CML-IA baseline 2013 & $\begin{array}{l}\text { Potentiel de pollution organique de l'eau } \\
\text { induit par l'introduction de nutriments } \\
\text { azotés et phosphatés dans les milieux } \\
\text { aquatiques. Dégradation de la qualité des } \\
\text { milieux et prolifération d'algues }\end{array}$ \\
\hline $\begin{array}{l}\text { Ecotoxicité des eaux } \\
\text { douces }\end{array}$ & USEtox & $\begin{array}{l}\text { Potentiel de toxicité apporté aux milieux } \\
\text { aquatiques (eaux douces de surface) } \\
\text { par l'émission de substances toxiques. } \\
\text { Déstabilisation et menace de la qualité et } \\
\text { de la variété des écosystèmes }\end{array}$ \\
\hline
\end{tabular}

a été construit à l'aide de la base de données Ecoinvent (v2.2) [9]. Cette base contient en effet un ICV de production de sucre intégrant la production de la betterave (phase agricole) et sa transformation en sucre. L'ICV de l'opération d'enrichissement par ajout de saccharose a été construit selon la méthode présentée préalablement pour l'enrichissement par MCQ, rétentat de RTS et OI i.e. collecte de données auprès d'experts. L'ICV économique est composé du coût d'achat du sucre (estimé par dire d'expert à $0,66 € / \mathrm{kg}$ ) et des coûts liés à l'utilisation au chai pour enrichir un moût, estimés en suivant la méthode employée pour les autres techniques.

L'enrichissement par ajout de MCR n'a pas pu faire l'objet d'un ICV environnemental en raison d'absence de données au moment de la réalisation de l'étude. L'ICV économique est construit selon le modèle employé pour l'enrichissement par ajout de saccharose : coût de l'achat du MCR estimé à 3,60 $€ / \mathrm{L}$ et coût liés à l'utilisation au chai pour enrichir, estimés par collecte de données auprès d'experts.

\subsection{Indicateurs d'impact environnementaux}

L'ACV est une méthode multi-critères i.e. qui exprime les dommages potentiels du système étudié sur la santé humaine, les écosystèmes ou encore les ressources naturelles. Pour l'expression de ces dommages, de nombreux indicateurs sont disponibles, définis à partir de méthodes caractérisation des impacts différentes. Pour le projet Enrichissement, les indicateurs ont été choisis en fonction de leur pertinence avec les enjeux environnementaux de la filière vitivinicole (cf. Tableau 2).

\section{Résultats}

Seuls les résultats obtenus pour l'enrichissement de $1 \mathrm{hl}$ de moût de $1 \%$ vol dans une cave produisant plus de 20000 hl située dans un bassin de production « sud » sont présentés ci-dessous. Les 10 scenarii étudiés sont l'ajout de MCQ blanc (achat et prestation), l'ajout de MCQ rouge (achat et prestation), l'ajout de rétentat de RTS (achat et prestation), l'ajout de saccharose, l'osmose inverse (achat et prestation) et l'ajout de MCR (volet économique uniquement).

\subsection{Impacts environnementaux potentiels}

Bien que s'appuyant sur la norme ISO 14040 [1], l'évaluation environnementale n'a pas fait intervenir de revue critique externe et indépendante, condition à la publication de résultats comparatifs d'études se réclamant ACV normée ISO 14040.

La Fig. 3 présente les impacts environnementaux potentiels. Pour chaque indicateur, l'impact de chaque scenario est exprimé par rapport au scenario le plus impactant, positionné à $100 \%$.

Les scenarii avec le modèle économique «Prestation » sont plus impactants que leur variante «Achat »sur les 4 indicateurs d'impact. La prestation entraîne en effet des transports de matières vers les sites de transformation (moût, MCQ) ou de matériels vers la cave (osmoseur, filtre ....).

Les scenarii d'enrichissement par ajout de MCQ (rouge ou blanc) sont les plus impactants sur les indicateurs de changement climatique, d'eutrophisation et d'écotoxicité aquatique. Pour l'enrichissement par ajout de MCQ blanc, les étapes du process les plus contributrices sont la filtration, l'électrodialyse et la concentration du moût. Pour le MCQ rouge, la thermovinification est également responsable d'une part importante des impacts. Ces étapes supposent une importante consommation d'électricité, d'eau et d'intrants de nettoyage, qui explique les impacts.

Les scenarii d'enrichissement par ajout de rétentat de RTS ont un impact important sur l'indicateur de Ressources (100\% pour le modèle en prestation et $82 \%$ pour le modèle achat). Cette technique consomme une quantité importante d'électricité pour réduire la teneur en sucre de la cuvée à dé-sucrer. Le rétentat obtenu ne permet pas d'enrichir autant que le MCQ (la concentration en sucre du rétentat est de $400 \mathrm{~g} / 1$ alors que celle du MCQ s'élève à $800 \mathrm{~g} / \mathrm{l}$ ). L'impact de la méthode est alors aussi expliqué par l'Unité Fonctionnelle choisie : enrichir $1 \mathrm{hl}$ de moût de $1 \%$ vol.

Le scenario d'enrichissement par ajout de saccharose présente des impacts faibles (inférieurs à $2 \%$ ) sur l'ensemble des indicateurs.

L'impact des scenarii d'enrichissement par osmose inverse est relativement faible sur l'ensemble des 


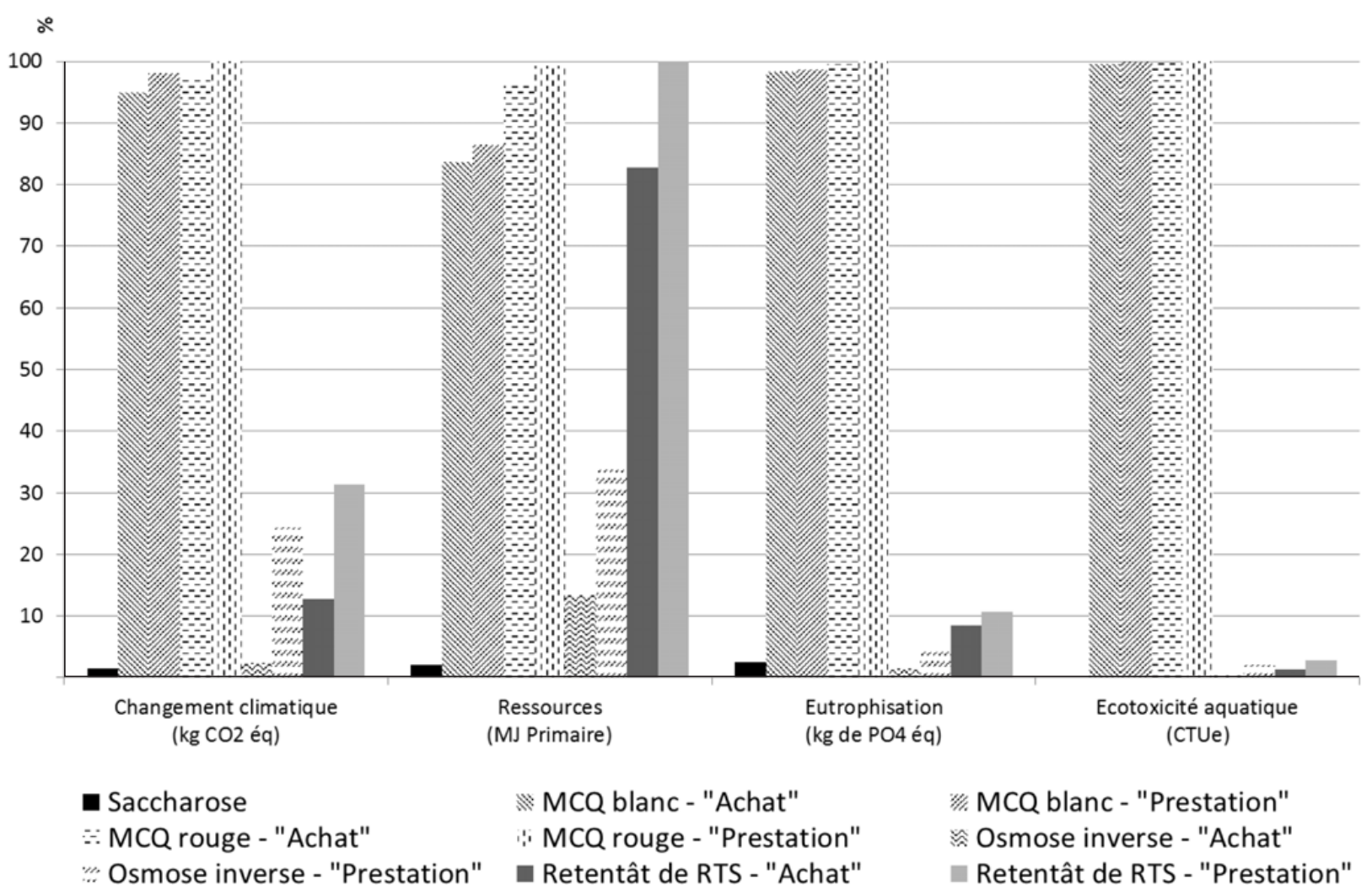

Figure 3. Impacts environnementaux potentiels comparés de l'enrichissement de $1 \%$ vol. d'1 hl de moût par différentes méthodes d'enrichissement, pour une cave produisant plus de $20000 \mathrm{hl}$, située dans un bassin de production 《sud ».

indicateurs, mis à part sur les Ressources et le Changement climatique pour le modèle prestation (respectivement $34 \%$ et $24 \%$ ), du fait des transports de matières et de matériels.

\subsection{Analyse économique}

La Fig. 4 présente, pour chaque scenario étudié, le coût de l'enrichissement d'1 hl de moût de $1 \%$ vol., et la répartition des coûts par poste de charge.

Les scenarii avec le modèle économique « achat» sont plus onéreux que leur variante «prestation ». Le prix des équipements nécessaires (électrodialyseur, concentrateur, osmoseur...), rapporté au volume de 200 hl de moûts enrichis, est en effet élevé.

Le scenario le plus onéreux est l'enrichissement par ajout de rétentat de RTS (modèle économique achat). Il s'élève à près de $100 €$ pour $1 \mathrm{hl}$ enrichi. L'achat de l'équipement est le principal contributeur au prix global.

Les scenarii d'enrichissement par ajout de MCQ (rouge et blanc) avec achat, ajout de rétentat de RTS en prestation et par osmose inverse avec achat s'élèvent à environ $20 €$ à $28 €$ pour 1 hl de moût enrichi.

Les scenarii MCQ (rouge et blanc) en prestation et ajout de MCR s'élèvent à environ $10 €$ à $12 €$ pour $1 \mathrm{hl}$ de moût enrichi.

Les scenarii d'enrichissement par osmose inverse en prestation et par ajout de saccharose sont les moins onéreux : environ $1,4 € /$ hl de moût enrichi.

Comme expliqué précédemment, l'une des hypothèses formulées pour la réalisation de cette étude est l'enrichissement en vue de l'obtention de 200 hl de moût enrichi. Cependant, on observe qu'à partir d'un certain volume de moût à enrichir, certains scenarii semblent plus pertinents (cf. Fig. 5).

L'enrichissement par ajout de saccharose reste le plus économique quel que soit le volume de moût traité. La courbe du scenario Osmose inverse - Achat est celle qui tend le plus vers celle du scenario Saccharose, signifiant qu'avec un volume important de moût à enrichir, l'acquisition de l'osmoseur est rapidement amortie.

\section{Discussion}

Les résultats sont influencés par la méthode choisie pour la construction des ICV et par les hypothèses formulées a priori.

Les ICV des deux nouvelles méthodes à l'étude ont été construits à partir de données collectées lors des expérimentations menées dans le cadre du volet faisabilité technique et organisationnelle du projet. Malgré la volonté de réalisation d'expérimentations en « grand volume», les contraintes règlementaires n'ont pas permis de mettre en œuvre des volumes de moûts supérieurs à 200 hl. Les installations mobilisées étaient alors configurées pour des volumes supérieurs. Les coûts et charges environnementales sont ainsi certainement surestimés. La Fig. 5 montre en effet que l'augmentation du volume traité diminue les coûts de traitement.

De plus, le caractère comparatif de l'étude nécessitant d'étudier chaque technique au sein d'une même cave, des caves-types ont été définies. Ce principe méthodologique influence la collecte de données qui ne peut pas être réalisée auprès d'un échantillon de caves reconnues 


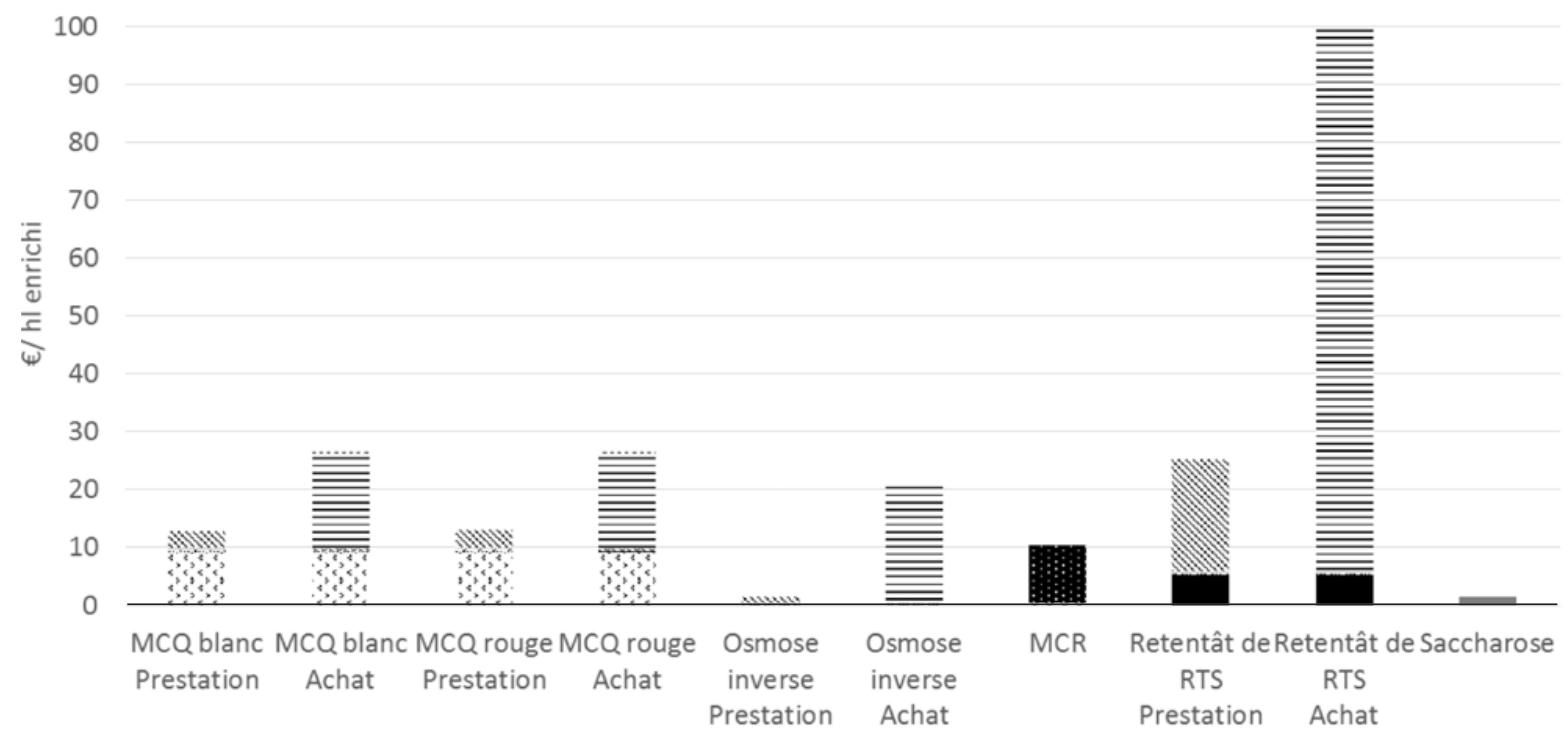
$\therefore$ Production du raisin
Intrants œnologiques
Main d'œuvre
III Intrants de nettoyage
Achat de saccharose

- Consommation électricité et eau

$z$ Transports

$\equiv$ Achat et maintenance équipements

NPrestations

Achat de MCR

Figure 4. Répartition des coûts de l'enrichissement de $1 \%$ vol. d'1 hl de moût par différentes méthodes d'enrichissement, pour une cave produisant plus de $20000 \mathrm{hl}$, située dans un bassin de production « sud ».

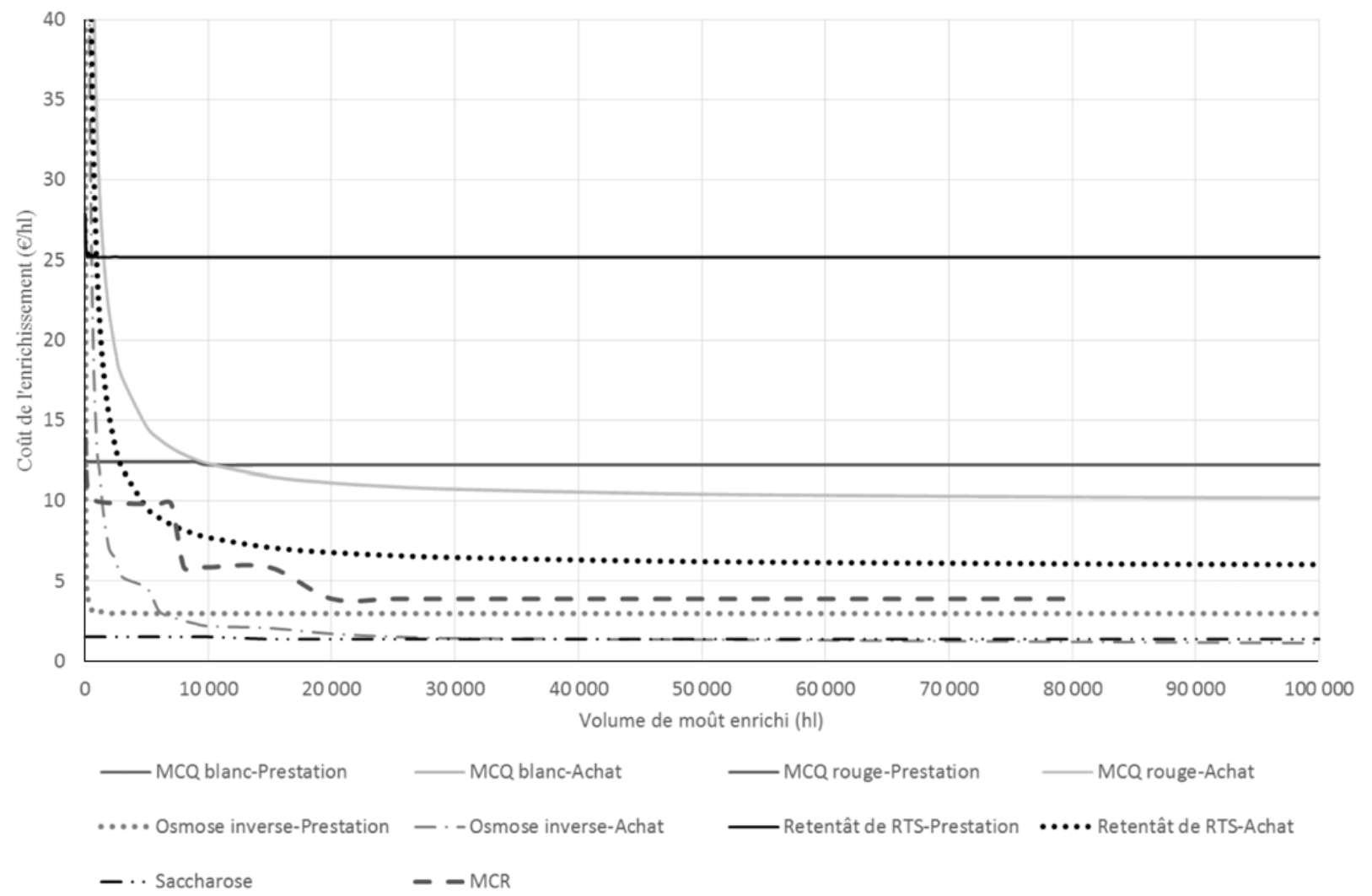

Figure 5. Coût de l'enrichissement de $1 \%$ vol. d' 1 hl de moût par différentes méthodes d'enrichissement, pour une cave produisant plus de 20000 hl, située dans un bassin de production «sud ». 
comme représentatives mais auprès d'experts formulant des hypothèses.

Le périmètre d'étude a été défini comme : de la fabrication du produit sucré à son utilisation pour enrichir. Il intègre ainsi la phase agricole : production des raisins et du moût transformé en MCQ, rétentat de RTS ou MCR ; production de la betterave transformée en saccharose. L'ICV de la phase viticole a été basé sur un itinéraire de production de raisins en AOP ou IGP. Les coûts de production des raisins pour l'élaboration du produit sucré sont alors élevés $(34 \%$ à $72 \%$ du coût global de l'enrichissement par ajout de MCQ pour une cave produisant plus de $20000 \mathrm{hl}$ dans un bassin « sud »). Il serait intéressant d'étudier une solution intégrant pour la phase viticole un vignoble dédié à l'élaboration du produit sucré, produisant des rendements élevés à de faibles coûts de production.

Cette solution pourrait être collective, permettant ainsi de réduire également les charges environnementales. En effet, l'hypothèse que chaque cave élabore son propre MCQ a été formulée. Les volumes mis en œuvre (40 hl de MCQ obtenus à partir de $200 \mathrm{hl}$ de moût) sont cohérents avec cette hypothèse. Cependant, l'augmentation du volume de MCQ produit par une même installation permettrait de réduire les charges environnementales du fait d'économies d'échelle sur les transports et les consommations d'énergie et d'eau.

La méthode de couplage des principes de l'ACV et de l'ACCV a permis d'étudier dans une perspective de comparaison des méthodes d'enrichissement additives et soustractives. Alors que les techniques additives (MCQ, rétentat de RTS, MCR, saccharose) augmentent le volume de moût enrichi (de 1,2 \% pour le saccharose à $8,5 \%$ pour le rétentat de RTS, selon les hypothèses formulées pour le projet Enrichissement), les techniques soustractives (osmose inverse) ont pour conséquence la diminution du volume de moût enrichi (de 8,6\% selon les hypothèses formulées pour le projet Enrichissement). L'utilisation de l'Unité Fonctionnelle « enrichir $1 \mathrm{hl}$ de $1 \%$ vol. » à laquelle se rapportent les résultats tant économiques qu'environnementaux, a permis de comparer les différentes techniques tout en prenant en compte les modifications de volume.

\section{Conclusion}

L'évaluation environnementale et économique de scenarii prospectifs pour l'enrichissement, comparés aux solutions existantes, est possible en suivant les principes et recommandations de l'ACV et de l'ACCV. Les résultats doivent cependant être analysés en ayant connaissance des hypothèses formulées, et des limites de la méthode employée pour la collecte des données environnementales et économiques.

La méthode définie pour les besoins du projet Enrichissement, ainsi que l'outil excel mis au point pour faciliter le traitement des données collectées, semblent pouvoir être utilisés pour l'évaluation environnementale et économique comparée, a priori, d'itinéraires de vinification, voire de tout itinéraire de transformation agroalimentaire. La prise en compte de l'amont agricole semble cependant plus difficile à mettre en œuvre.

Les résultats présentés sont issus d'une collecte de données effectuée en 2015. Ils reflètent les prix observés au cours de ce millésime (coûts d'achat du saccharose, du MCR, montant des prestations d'électrodialyse, concentration...). Si des tensions devaient apparaître sur certains marchés, le fait de produire soit même son produit sucré et de pouvoir le stocker d'une année sur l'autre pourrait engendrer une diminution de la dépendance des entreprises vinicoles aux fluctuations des cours du marché.

\section{Références}

[1] ISO, ISO 14040 - environmental management - life cycle assessment - principles and framework (2006)

[2] G. REBITZER, "Integrating Life Cycle Costing and Life Cycle Assessment for Managing Costs and Environmental Impacts in Supply Chains", Cost Management in Supply Chains (2002)

[3] Règlement (CE) n ${ }^{\circ}$ 479/2008 du Conseil du 29 avril 2008, Annexe V

[4] Règlement (CE) $\mathrm{n}^{\circ} 555 / 2008$ du 27 juin 2008, article 33

[5] Règlement (CE) n ${ }^{\circ}$ 479/2008 du Conseil du 29 avril 2008, Annexe IV

[6] IFV, Techniques soustractives d'enrichissement des moûts, Cahier Itinéraire n 14 (2007)

[7] P. Koch, T. Salou, V. Colomb, S. Payen, S. Perret, A. Tailleur, S. Willmann, Agribalyse: rapport méthodologique, Version 1.2 (2015)

[8] FranceAgriMer, CER France, Observatoire viticole 2009-2010 (2012)

[9] R. Frischknecht, N. Jungbluth, H.-J. Althaus, G. Doka, R. Dones, T. Heck, S. Hellweg, R. Hischier, T. Nemecek, G. Rebitzer and M. Spielmann, The ecoinvent database: Overview and methodological framework, International Journal of Life Cycle Assessment, Vol. 10, 3-9 (2005) 